\title{
Buckling of cylindrical sandwich shells with metal foam cores
}

\author{
J.W. Hutchinson ${ }^{\mathrm{a}, *}$, M.Y. He ${ }^{\mathrm{b}}$ \\ ${ }^{a}$ Division of Engineering and Applied Sciences, Harvard University, 315, Pierce Hall, 29 Oxford Street, Cambridge, MA 02138, USA \\ b Materials Engineering Department, University of California, Santa Barbara, CA 93106, USA
}

Received 10 November 1999

\begin{abstract}
Buckling of cylindrical sandwich shells subject to axial compression is addressed for shells having foamed metal cores. Optimal face sheet thickness, core thickness and core density are obtained which minimize the weight of a geometrically perfect shell with a specified load carrying capacity. Constraints imposed by wrinkling and yielding of the face sheets and yielding of the core are all considered. The range of the structural load index is identified for which the sandwich shells have a competitive weight advantage over stringer stiffened shells. In most of this range, the minimum weight design has elastic buckling simultaneous with face sheet yielding. Imperfection sensitivity of the shells is assessed with special emphasis on the role of plasticity in degrading strength, especially in light of the coincidence of elastic buckling and face sheet yielding in the optimally designed perfect shell. The purpose is to examine the interaction between imperfections and plastic yielding to see if buckling load knockdowns should be larger than those expected for elastic shells. (c) 2000 Elsevier Science Ltd. All rights reserved.
\end{abstract}

Keywords: Buckling; Cylindrical sandwich shells; Metal foams; Imperfections

\section{Introduction}

Economical processes to produce metal foams have advanced to the point where applications are being explored (Evans et al., 1998). Application as cores for sandwich plates and shells is considered promising for a number of reasons. Face sheets can be integrally formed with a closed cell core producing sandwiches without the usual environmental and delamination susceptibilities of honeycomb sandwiches. Methods are being developed which permit sandwich components to be curved to form a shell prior to foaming the core. If successful, such methods would side step the difficult and costly process of assembling a sandwich shell by joining precurved face sheets and core. Integrally formed metal foam core sandwich panels may also have thermal and acoustic advantages over the conventional panel structure.

This article addresses two issues related to axially loaded cylindrical sandwich shells with metal foam cores. The first is to identify the load range for which the sandwich shells have a competitive weight advantage over shells of a more conventional construction. The second is to examine the interaction between

\footnotetext{
${ }^{*}$ Corresponding author. Tel.: +1-617-495-2848; fax: +1-617-495-9837.

E-mail address: hutchinson@husm.harvard.edu (J.W. Hutchinson).
} 
imperfections and plastic yielding in optimally designed sandwich shells to see if larger buckling load knockdowns than those occurring for elastic cylindrical shells are to be expected.

Agarwal and Sobel (1977) established the weight competitiveness of a honeycomb sandwich construction for cylindrical shells under axial compression compared to the more established construction involving rings and stiffeners. By comparing weight optimized shells, these authors showed that honeycomb sandwiches offer a substantial weight advantage over a significant load range. It does not follow from this finding that metal foamed core sandwiches will necessarily have a similar competitive weight advantage because the relative core weight of these sandwiches is much greater than that for honeycomb construction. Indeed, Budiansky (1999) has found that flat sandwich panels with metal foam cores generally do not have a potential weight advantage over their axially stiffened counterparts for the case of uniaxial loads. There is, however, no benefit derived from the equi-biaxial stretching and bending stiffnesses of the sandwich construction in the case of a uniaxially loaded flat panel, whereas, the biaxial stiffnesses do contribute to load carrying capacity in most shell buckling problems. Thus, it will be seen that there is an important range of loads for which optimized sandwich construction employing cores of metal foam have a distinct weight advantage over the lightest weight metal stringer construction for cylindrical shells loaded in axial compression.

The uniaxially compressed sandwich shells will be optimized under the assumption that they are free of imperfections. The strong buckling sensitivity of this class of structures to geometric imperfections is usually taken into account by use of a knock-down factor multiplying the buckling load of the perfect shell, thereby reducing the estimated load carrying capability to a level deemed appropriate for shells of a given radius to thickness ratio (NASA, 1965). Knock-down factors have been established empirically using a large but diverse set of experimental buckling loads obtained for shells which are designed to buckle in the elastic range. The motivation for the present imperfection-sensitivity study stems from the fact that optimized perfect sandwich shells turn out to have face sheet yielding coincident with overall buckling over most of the load range in which they have a weight advantage. Thus, the question arises as to whether interaction between imperfections and plastic yielding will require larger knock-down factors for these shells. The starting point in the present analysis is the elastic imperfection-sensitivity analysis of Koiter (1963) as extended by Tennyson and Chan (1990) to sandwich cylindrical shells under axial compression. The onset of plastic yielding in the imperfect shell is determined, and it is shown that in most instances buckling precedes yielding if the face sheet yield stress has been designed such that the perfect shell has coincident yielding and elastic buckling. A numerical finite element model is also developed and employed to address the interaction between imperfections and plastic yielding in the optimized shells.

The face sheets and the core are each taken to be isotropic. The face sheets have Young's modulus $E_{\mathrm{f}}$, Poisson's ratio $v_{\mathrm{f}}$, compressive yield stress $\sigma_{\mathrm{Y}}$, weight density $\rho_{\mathrm{f}}$, and thickness $t$. The thickness of the core is denoted by $d$. With the weight density of the fully dense core metal specified by $\rho_{\mathrm{c}}^{0}$, the density of the metal foam is given by $\rho_{\mathrm{c}}=\eta \rho_{\mathrm{c}}^{0}$, where $\eta$ will be referred to as the relative density of the core. Following Gibson and Ashby (1997) and Sugimura et al. (1997), the relation between Young's modulus of the core material, $E_{\mathrm{c}}$, and the relative density can be written as

$$
E_{\mathrm{c}} / E_{\mathrm{c}}^{0}=\alpha \eta^{2}
$$

where $E_{\mathrm{c}}^{0}$ is the modulus of the fully dense material and $\alpha$ is a numerical coefficient chosen to give the best fit to experimental modulus data over the range of $\eta$ of interest. Current manufacturing processes for metal foams lead to $\alpha \cong 1$ for relative densities centered about $\eta=0.1$. If improvements in material morphology can be made, there is reason to hope that modulus levels might be increased to levels consistent with $\alpha \cong 4$. A limited investigation of the influence of this coefficient will be made. With $v_{\mathrm{c}}$ as Poisson's ratio of the metal foam, the shear modulus of the core is $G_{\mathrm{c}}=E_{\mathrm{c}} /\left[2\left(1+v_{\mathrm{c}}\right)\right]$. The compressive yield stress of the core foam is denoted by $\sigma_{\mathrm{Y}}^{\mathrm{c}}$. 


\section{Buckling of perfect axially compressed sandwich shells}

\subsection{Elastic overall buckling of perfect shell}

A perfect cylindrical shell of radius $R$ is considered subject to a uniform axial compressive force per unit length, $N$, such that the total force is $P=2 \pi R N$. When end conditions are ignored, the classical elastic buckling problem admits sinusoidal mode shapes which are periodic in the axial and circumferential directions. Buckling (Zahn and Kuenzi, 1963; Sullins et al., 1969; Tennyson and Chan, 1990) takes place in an axisymmetric mode with a short axial wavelength which is proportional to $\sqrt{R t_{\text {eff }}}$, where $t_{\text {eff }}=\sqrt{3} d$ is the effective shell thickness. Unlike the monocoque isotropic cylindrical shell, the buckling eigenvalues associated with non-axisymmetric modes are appreciably higher than that of the critical axisymmetric mode if the shear stiffness of the core is low. A synopsis of the elastic buckling analysis is given in Appendix A. The critical $N$ for axisymmetric buckling is

$$
\frac{N}{E_{\mathrm{f}} R}=\frac{2 t d}{\sqrt{1-v_{\mathrm{f}}^{2}} R^{2}}\left[1-\frac{\mu}{\sqrt{2}}\right],
$$

where

$$
\mu=\frac{E_{\mathrm{f}} t}{\sqrt{2\left(1-v_{\mathrm{f}}^{2}\right)} G_{\mathrm{c}} R} .
$$

This result applies if the length of the shell $L$ is at least several times the axial buckle wavelength. The applicability of this result also rests on the assumption that sufficiently strong end support conditions are in effect. End conditions modify Eqs. (2.1a) and (2.1b) slightly, but not nearly as much as initial imperfections which will be considered in Sections 4 and 5. Thus, Eqs. (2.1a) and (2.1b) are taken as the buckling criterion for the perfect shell.

Formula (2.1a) has been simplified assuming $d$ is large compared to $t$, consistent with optimally designed sandwich shells considered in Section 3. Thus, the bending stiffness of each face sheet about its own midplane can be neglected with little error. Additionally, the load carrying contribution of the core and its contribution to the bending stiffness have been neglected in this formula. (The core shear stiffness is not neglected.) As a consequence, the buckling load of the perfect shell is underestimated, but the error is small when the elastic modulus of the core is sufficiently low compared to that of the face sheets (i.e. $\left.E_{\mathrm{c}} / E_{\mathrm{f}} \ll 2 t / d\right)$. Under these conditions, the primary function of the core is to maintain the separation of the face sheets, which carry the axial load, and to provide an adequate shear stiffness to carry transverse shear forces in the shell when non-uniform deformations develop. When $\mu$ defined in Eq. (2.1b) is small, there will be relatively little reduction in the buckling load due to core shear compliance. Moreover, the validity of Eq. (2.1a) requires that $\mu<1 / \sqrt{2}$, ensuring that localized shear kinking is excluded. This condition will be imposed on the shells considered. The finite element model of the shell employed in Section 6 accounts for the contributions to the bending and stretching stiffnesses, verifying the small errors involved in making these approximations in the analytical part of the study.

\subsection{Yield of face sheets and core, and face sheet wrinkling}

With $\sigma_{\mathrm{Y}}$ as the compressive yield stress of the face sheets, the condition for face sheet yielding of the perfect shell prior to, or simultaneous with, buckling is

$$
N=2 t \sigma_{\mathrm{Y}} \text {. }
$$

Yielding of the core does not directly affect the load carrying capacity as the core is assumed to support no load. However, core yielding will affect the ability of the core to suppress face sheet wrinkling and to 
maintain initial postbuckling load carrying capacity. Yielding of the core prior to buckling in the perfect shell will be avoided if the axial strain in the unbuckled shell, $\varepsilon=N /\left(2 t E_{\mathrm{f}}\right)$, does not exceed the uniaxial compressive yield strain of the metal foam, $\sigma_{\mathrm{Y}}^{\mathrm{c}} / E_{\mathrm{c}}$. In other words, core yielding is excluded if the yield strain of the core is higher than that of the face sheets. If neither the core nor the face sheets have yielded, face sheet wrinkling is governed by the condition that the compressive stress in the face sheet attain $B\left(E_{\mathrm{f}} E_{\mathrm{c}}^{2}\right)^{1 / 3}$, where $B$ is a coefficient given by Allen (1969) which is approximately 0.58 . The condition assumes that the core is sufficiently thick and that there is no interaction with the opposite face sheet. In terms of the compressive resultant stress, the onset of face wrinkling in the perfect shell occurs when

$$
N=2 t B\left(E_{\mathrm{f}} E_{\mathrm{c}}^{2}\right)^{1 / 3} .
$$

\section{Optimally designed perfect sandwich shells}

Optimum configurations are sought for a cylindrical shells of prescribed radius $\mathrm{R}$ and length $L$ that are designed to carry a prescribed load $P$, or, equivalently, a prescribed load per circumferential length, $N=P /(2 \pi R)$. The face sheet material is specified, and it is assumed that the fully dense base metal of the foam in the core is the same as the face sheet material such that $E_{\mathrm{f}}=E_{\mathrm{c}}^{0} \equiv E$ and $\rho_{\mathrm{f}}=\rho_{\mathrm{c}}^{0} \equiv \rho$. The class of metal foams characterizing the core is taken to be specified in the sense that $\alpha$ in Eq. (1.1) is specified. Two optimization problems are posed. In the first, the relative density $\eta$ of the core is also taken to be specified, while thicknesses of the face sheets $t$ and core $d$ are sought that give rise to the minimum weight shell, subject to the condition that $N$ exceeds none of Eqs. (2.1a)-(2.3). In the second, termed the global optimum, the relative core density $\eta$ is treated as a variable in the minimization process along with $t$ and $d$.

The weight of the shell is

$$
W=2 \pi R L \rho(2 t+\eta d) .
$$

The auxiliary function used to find the variables characterizing the minimum weight shell is

$$
F(t, d, \eta)=W-\lambda_{1}\left[N-\frac{2 E t d / R}{\sqrt{1-v^{2}}}\left[1-\frac{4 \eta^{-2} t / R}{3 \alpha \sqrt{1-v^{2}}}\right]\right]-\lambda_{2}\left[N-2 t \sigma_{\mathrm{Y}}\right]-\lambda_{3}\left[N-2 B E \alpha^{2 / 3} t \eta^{4 / 3}\right] .
$$

The core shear modulus has been expressed in terms of the relative core density using Eq. (1.1) and $v_{\mathrm{c}}=1 / 3$. Poisson's ratio $v$ in Eq. (3.2) is that of the face sheets. Three Lagrangian multipliers, $\lambda_{i}$, have been introduced to enforce the constraints, Eqs. (2.1a)-(2.3), when they are active. The optimum sought is that combination of variables with minimizes $F$ subject to the constraints. The constraint associated with overall buckling is always active because it is the only one of the three depending on $d$. If one or more of the constraints is not active, the corresponding term in Eq. (3.2) is dropped.

\subsection{Optimal shells with $\eta$ prescribed}

If the core density is chosen such that $\eta>\left(\sigma_{\mathrm{Y}} / E B \alpha^{2 / 3}\right)^{3 / 4}$, face sheet wrinkling will be excluded as a possibility because face sheet yielding will always intervene first. Assuming this is the situation, there are two possibilities: (i) optimum weight is attained for a shell which buckles in the elastic range, or (ii) elastic buckling and face sheet yielding occur simultaneously. In the case where face sheet yielding is not active, combinations $(W, N)$ at the optimum are generated by regarding $t$ as a free variable. The associated value of $d$ is given by

$$
d / R=\frac{2\left[a_{1}-a_{2}(t / R) \eta^{-2}\right](t / R)}{\eta\left[a_{1}-2 a_{2}(t / R) \eta^{-2}\right]}
$$


with $(W, N)$ then evaluated from Eqs. (3.1) and (2.1a). Here, and throughout this article,

$$
a_{1}=2 / \sqrt{1-v^{2}}, \quad a_{2}=8 /\left[3 \alpha\left(1-v^{2}\right)\right] .
$$

These results are only valid in the range of $t$ for which $N<2 t \sigma_{\mathrm{Y}}$. When face sheet yielding (2.2) is active at the optimum,

$$
t=\frac{N}{2 \sigma_{\mathrm{Y}}}, \quad \frac{d}{R}=\frac{N}{E R}\left[a_{1} t / R-a_{2}(t / R)^{2} \eta^{-2}\right]^{-1}
$$

and $W$ is obtained from Eq. (3.1).

Fig. 1a presents the weight index, $W /\left(2 \pi R^{2} L \rho\right)$, as a function of the load index, $N /(E R)$, for the optimum shells for two values of the relative core density and a yield stress representative of an aluminum structural alloy, $\sigma_{\mathrm{Y}} / E=0.007$. The curves for the sandwich shells are independent of the length of the shell, $L$. For
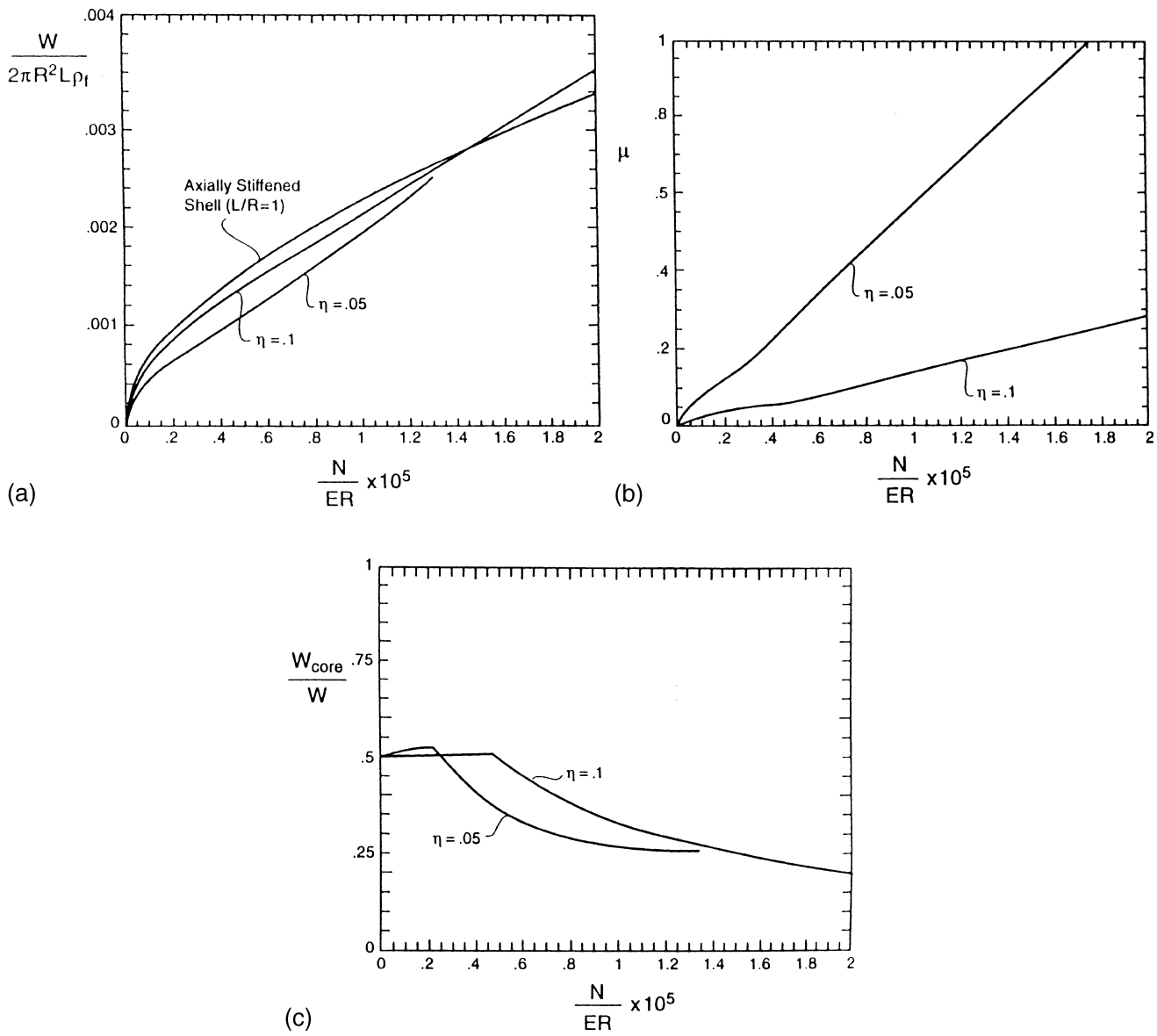

Fig. 1. (a) Weight index versus load index for metal foam core cylindrical sandwich shells under axial compression. For each value of the load index, the shell's face sheet and core thicknesses have been chosen to minimize its weight at prescribed values of the relative core density $\eta$. The curve for the axially stiffened cylinder applies to an optimized hat-stiffened shell buckling between rings spaced a distance equal to the shell radius. (b) The parameter $\mu$ which measures the shear compliance of the core. (c) Ratio of core weight to total weight $\left(\alpha=1, \sigma_{\mathrm{Y}} / E_{\mathrm{f}}=0.007, E_{\mathrm{c}}^{0}=E_{\mathrm{f}}, v_{\mathrm{f}}=v_{\mathrm{c}}=1 / 3\right)$. 
values of the load index above about $0.4 \times 10^{-5}$, face sheet yielding coincides with overall elastic buckling. Below this, overall elastic buckling is the sole determinant of strength. The parameter $\mu$ defined in Eq. (2.1b) is plotted in Fig. 1b. A small value of $\mu$ pertains to the shell having the higher core density, $\eta=0.1$, implying that the compliance of the core does not significantly reduce the buckling load below that for a shell with a very stiff core. The lower density core, however, is associated with higher values of $\mu$. Only for the load index less that $1.2 \times 10^{-5}$ does the optimal solution above meet the condition $\mu<1 / \sqrt{2}$, which excludes localized shear kinking. At higher loads, the constraint $\mu \leqslant 1 / \sqrt{2}$ would be activated. The ratio of the weight in the core to the total weight of the shell for the shells is plotted in Fig. 1c. In the middle of the range of the load index, the core comprises about $25 \%$ the total weight of the shell. This is far below the relative core weight predicted for the globally optimized elastic shell described later in this section.

These results in Fig. 1 have been plotted over the range of indices for which foam metal core sandwich cylinders have a competitive advantage over conventional stiffened shells. This assertion is illustrated by the curve for a cylindrical shell of the same aluminum alloy with axial hat-shaped stiffeners which are located on the inside of the shell. This shell has also been weight optimized subject to the prescribed $N$. Unlike the sandwich shells, the weight index for the optimum stiffened shell depends on the shell length, and the results shown are for a shell whose buckle half-wavelength $L$ in the axial direction is equal to $R$. This would represent the case of a shell buckling between rings with a relatively low torsional stiffness spaced a distance $R$ apart. Larger spacings would require a higher weight index and vice versa. A lower weight index is obtained if the hat stiffeners are located on the outside of the cylinder rather than the inside, but outside placement is often excluded for reasons having nothing to do with structural efficiency. The optimized axially stiffened shell in Fig. 1 buckles in the elastic range for all values of the load index for which results are plotted.

\subsection{Globally optimal shells with a core density $\eta$ as a variable in the weight minimization}

Optimization with $t, d$ and $\eta$ as variables in the weight minimization process leads to the four possibilities given below.

\subsubsection{Overall buckling such that face sheet yielding and wrinkling do not occur}

Index pairs $(W, N)$ are generated from Eqs. (2.1a), (2.1b) and (3.1) for each $\eta$, where $t$ and $d$ are given by

$$
\frac{d}{R}=\frac{4 a_{1} \eta}{3 a_{2}}, \quad \frac{t}{R}=\frac{a_{1} \eta^{2}}{3 a_{2}}
$$

It is readily verified that the elastic, globally optimized shell has two thirds of its weight in the core. However, designs that account for yielding and wrinkling have significantly lower relative core weight in the load range for which the sandwich cylinders are competitive. For Eq. (3.6) to hold, the load index must be such that face sheet yielding and wrinkling do not occur. Explicit expressions for the indices are

$$
\frac{W}{2 \pi R^{2} L \rho}=\frac{2 a_{1} \eta^{2}}{a_{2}}, \quad \frac{N}{E R}=\frac{8 a_{1}^{3} \eta^{3}}{27 a_{2}^{2}} .
$$

The relative reduction of the buckling load due to the shear compliance of the core is found to be precisely $1 / 3$ for shells optimized against elastic buckling, i.e. in Eqs. (2.1a) and (2.1b)

$$
\mu=\frac{E_{\mathrm{f}} t}{\sqrt{2\left(1-v_{\mathrm{f}}^{2}\right)} G_{\mathrm{c}} R}=\frac{\sqrt{2}}{3} .
$$

In the terminology of Tennyson and Chan (1990), such shells have moderately stiff cores, and Eq. (3.8) ensures that localized shear kinking mode is excluded. 


\subsubsection{Overall buckling with face sheet yielding but no wrinkling}

The weight (3.1) is generated in terms of $N$ and the relations

$$
\eta=\sqrt{\frac{3 a_{2} N}{2 a_{1} \sigma_{\mathrm{Y}} R}}, \quad \frac{t}{R}=\frac{a_{1} \eta^{2}}{3 a_{2}}, \quad \frac{d}{R}=\frac{N}{E R}\left[a_{1} t / R-a_{2}(t / R)^{2} \eta^{-2}\right]^{-1}
$$

assuming that the condition for face sheet wrinkling is not violated.

\subsubsection{Overall buckling with face sheet wrinkling but no yielding}

The relative core density $\eta$ is obtained in terms of $N$ from the non-linear equation

$$
\frac{N}{E R}\left[2 X^{2} \eta^{-1 / 3}+5 a_{2} B \alpha^{2 / 3}\right]-7\left(B \alpha^{2 / 3}\right)^{2} X \eta^{10 / 3}=0, \quad \text { where } X=a_{1}-\frac{a_{2} N \eta^{-10 / 3}}{2 B \alpha^{2 / 3} E R} .
$$

Then, $t$ and $d$ are given by

$$
\frac{t}{R}=\frac{N \eta^{-4 / 3}}{2 B \alpha^{2 / 3} E R}, \quad \frac{d}{R}=\frac{N}{E R}\left[a_{1} t / R-a_{2}(t / R)^{2} \eta^{-2}\right]
$$

and $W$ is obtained from Eq. (3.1). Now, face sheet yielding must not occur.

\subsubsection{Overall buckling with face sheet yielding and wrinkling}

In this case,

$$
\eta=\left(\frac{\sigma_{\mathrm{Y}}}{B \alpha^{2 / 3} E}\right), \quad \frac{t}{R}=\frac{N}{2 \sigma_{\mathrm{Y}} R}, \quad \frac{d}{R}=\frac{N}{E R}\left[a_{1} t / R-a_{2}(t / R)^{2} \eta^{-2}\right]^{-1}
$$

with $W$ given by Eq. (3.1).

The weight index of the globally optimized shell is plotted in Fig. 2a over the same range of the load index used in the earlier plot, for the same value of the face sheet yield stress, $\sigma_{\mathrm{Y}} / E=0.007$. The upper of
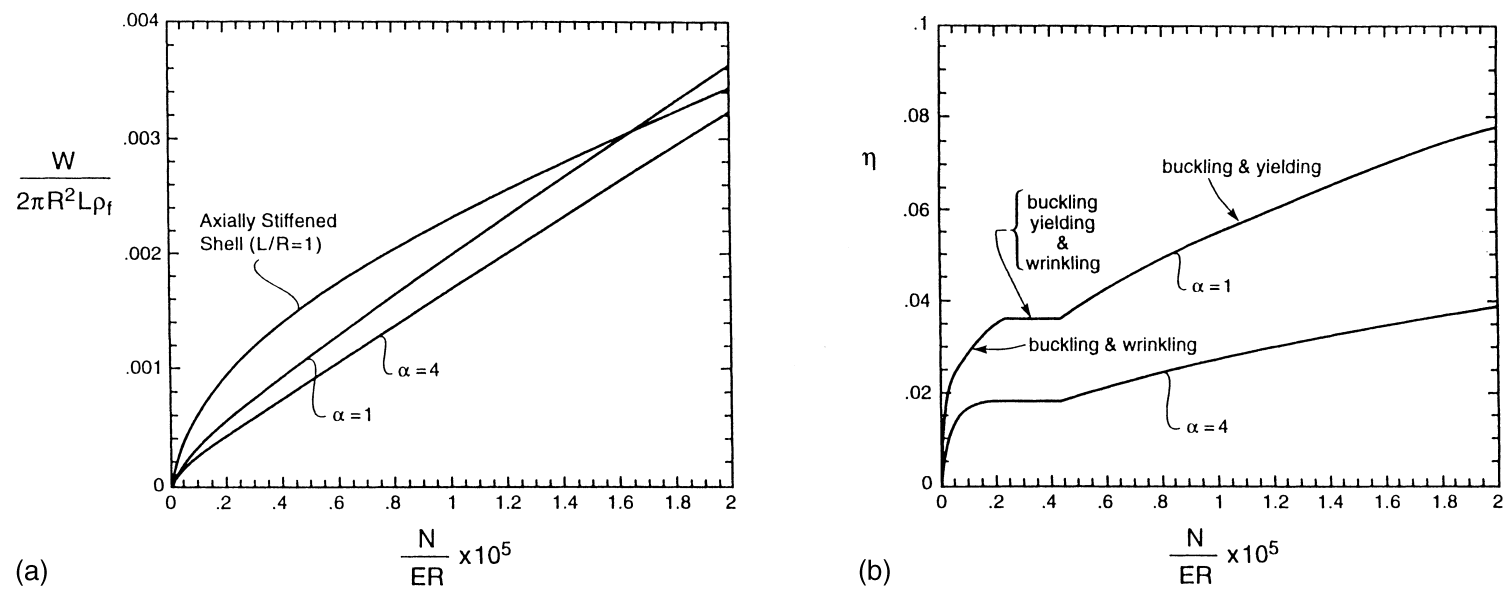

Fig. 2. (a) Weight index versus load index for globally optimized metal foam core cylindrical sandwich shells under axial compression. For each value of the load index, the shell's face sheet thickness, core thickness, and relative density have been chosen to minimize its weight. Results are shown for two values of the coefficient $\alpha$ which determines the elastic shear stiffness of the metal foam in the core. The curve for the axially stiffened cylinder applies to an optimized hat-stiffened shell buckling between rings spaced a distance equal to the shell radius. (b) Values of the relative core density $\eta$, and identification of the operative constraints for the optimized shell $\left(\sigma_{\mathrm{Y}} / E_{\mathrm{f}}=0.007, E_{\mathrm{c}}^{0}=E_{\mathrm{f}}, v_{\mathrm{f}}=v_{\mathrm{c}}=1 / 3\right)$. 
the two curves for the sandwich shells for $\alpha=1$ corresponds to a foam manufactured by current standards (cf. Eq. (1.1)). The same value of $\alpha$ was used in computing the results in Fig. 1. The lower of the two curves $(\alpha=4)$ illustrates the improved weight performance that can be expected from a foam with better stiffness properties. The relative core density is shown in the accompanying plot (Fig. 2b). In this plot, the constraints operating at each value of the load index are indicated. At the lowest values of the index overall buckling is simultaneous with face sheet wrinkling, at intermediate values overall buckling occurs with both face sheet yielding and wrinkling, whereas at the highest levels overall buckling occurs simultaneously with face sheet yielding. This sequence follows the same trends Budiansky (1999) found for optimized flat sandwich panels with foamed metal cores. The condition, $\mu<1 / \sqrt{2}$, is met for all the shells in Fig. 2 .

The curve for the optimized axially stiffened cylindrical shell from Fig. 1 is replotted in Fig. 2. On the basis of the results for perfect shells, one concludes that foamed metal sandwich cylinders have a potential weight advantage of as much as a factor of two over their optimized axially stiffened counterparts in the lower range of the load index. As mentioned earlier, Agarwal and Sobel (1977) established the weight advantage of honeycomb sandwich cylindrical shells over axially stiffened cylinders, but honeycomb cores are lighter than foamed metal cores and, therefore, constitute a smaller fraction of the total shell weight. The fact that the foamed metal core sandwich shells have superior performance to axially stiffened shells at low values of the load index can be attributed to their biaxial bending and stretching stiffnesses.

\section{Influence of imperfections on optimally designed shells}

Sandwich shells designed optimally according to the specifications derived for the perfect shells in the last section will be considered from the point of view of their sensitivity to geometric imperfections. Cylindrical shells under axial compression are notorious for their buckling sensitivity. The load reduction due to imperfections is commonly addressed by use of a multiplicative knock-down factor on the buckling load of the perfect shell (NASA, 1965). The factor for cylindrical shells with large radius to thickness ratios can be as small as $1 / 4$ or even $1 / 5$. The question posed in this section is whether the optimally designed shells are unduly imperfection sensitive due to the fact that buckling and plastic yielding occur simultaneously over most of the load index range of interest for the perfect realizations. In other words, do geometric imperfections interact with plastic yielding to lower the buckling strength of the shells to a larger extent than one would expect for shell designed to buckle elastically? Fortunately, the answer to the question appears to be that they do not, at least not significantly, as the results which follow reveal.

Structures are most susceptible to imperfections in the shape of their buckling modes (Koiter, 1945). For the cylindrical sandwich shells, for which the critical buckling mode is axisymmetric, an axisymmetric imperfection in the form of an initial normal displacement of the shell middle surface is considered:

$$
\bar{w}=\bar{\xi} \cos \left(\lambda x_{1} / R\right),
$$

where $\bar{\xi}$ is the imperfection amplitude. Subsequently, $\lambda$ will be taken as $\lambda_{\mathrm{c}}$, corresponding to the wave number of the critical mode, cf. Eq. (A.16). The thicknesses of the core and face sheets are unchanged from the values for the perfect shell. For geometric imperfections of the form (4.1), the shell equations (cf. Appendix A, Eqs. (A.1)-(A.8)) admit an exact, closed form axisymmetric solution, as originally produced for the monocoque shell by Koiter (1963) and as provided for sandwich shells by Tennyson and Chan (1990). Algebraic steps in the solution process will be omitted here. Only solution quantities needed to evaluate the onset of plastic yielding in the face sheets and the core will be presented.

As before, let $N^{0}$ be the applied compressive load per circumferential length. Let $N_{\mathrm{c}}^{0}(\lambda)$ denote the eigenload $N^{0}$ defined by Eq. (A.14) in Appendix A with $\lambda=\lambda_{1}$ and $\lambda_{2}=0$. Note that with $\lambda=\lambda_{\mathrm{c}}, N_{\mathrm{c}}^{0}(\lambda)$ becomes Eq. (2.1a). The additional normal displacement of the shell middle surface is 


$$
w=\frac{\bar{\xi} N^{0}}{N_{\mathrm{c}}^{0}(\lambda)-N^{0}} \cos \left(\lambda x_{1} / R\right)
$$

Tennyson and Chan (1990) investigated non-axisymmetric bifurcation from this axisymmetric state, following the approach used by Koiter (1963) in his famous study of monocoque cylindrical shells under axial compression. At small imperfection levels, the bifurcation load coincides with the maximum load the shell can support. The results of Koiter and Tennyson and Chan are presented in Fig. 3 as the ratio of the buckling load of the imperfect shell to that of the perfect shell, $\left(N_{\mathrm{c}}^{0}\right)_{\mathrm{imp}} / N_{\mathrm{c}}^{0}$, as a function of a normalized imperfection amplitude. In the case of the monocoque shell, the imperfection amplitude $\bar{\xi}$ is normalized by the shell thickness, $t$, whereas, for the sandwich shells it is normalized by an effective thickness defined by

$$
t_{\text {eff }}=\sqrt{3} d \text {. }
$$

A sandwich shell with a stiff core $(\mu \ll 1 / \sqrt{2})$ is equivalent to a monocoque shell with effective thickness (4.3) and effective modulus $E_{\text {eff }}=2\left(t / t_{\text {eff }}\right) E_{\mathrm{f}}$.

The choice of Eq. (4.3) necessarily brings the buckling loads of the imperfect sandwich shells with small values of $\mu$ into coincidence with those of the monocoque shell. It is evident from Fig. 3 that Eq. (4.3) remains successful in this respect even for shells with relatively compliant cores, although there is some amelioration of the knock-down from the imperfection as the core becomes more compliant. Thus, there appears to be a strong case for taking $R /(\sqrt{3} d)$ as the "radius to thickness ratio" of the sandwich shell in the determination of the buckling knock-down factor from the large experimental data set collected by NASA (1965).

\subsection{Onset of yielding in the face sheets of the imperfect shell}

Now, the effect of the imperfection on the stresses in the face sheets is investigated. Denote the axial stress in each face sheet in the perfect shell at the eigenload, $N^{0}=N_{\mathrm{c}}^{0}(\lambda)$, by $\sigma_{11} \equiv-\sigma_{\mathrm{c}}(\lambda)=-N_{\mathrm{c}}^{0}(\lambda) /(2 t)$. The stresses in the outer face sheet of the imperfect shell can be obtained from the same axisymmetric solution. They are given by

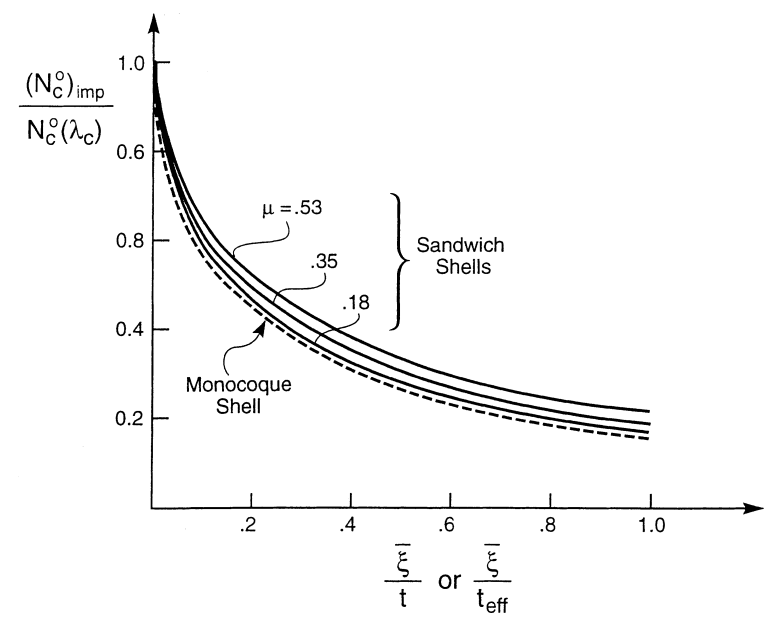

Fig. 3. Ratio of the elastic buckling load of the imperfect shell to that of the perfect shell as a function of the amplitude of the axisymmetric imperfection, $\bar{\xi}$. For Koiter (1963) the result for the monocoque shell, the imperfection amplitude is normalized by the shell thickness, $t$. For the results of Tennyson and Chan (1990) for sandwich shells, the imperfection amplitude is normalized by the effective thickness, $t_{\text {eff }}$, defined by Eq. (4.3). 


$$
\begin{aligned}
& \frac{\sigma_{11}^{+}}{\sigma_{\mathrm{c}}(\lambda)}=\frac{N^{0}}{N_{\mathrm{c}}^{0}(\lambda)}\left\{-1+\frac{\sqrt{3} b^{2}}{1+\mu b+b^{2} / 2} \frac{\left(\bar{\xi} / t_{\mathrm{eff}}\right)}{\left[1-N^{0} / N_{\mathrm{c}}^{0}(\lambda)\right]} \cos \left(\lambda x_{1} / R\right)\right\}, \\
& \frac{\sigma_{22}^{+}}{\sigma_{\mathrm{c}}(\lambda)}=\frac{N^{0}}{N_{\mathrm{c}}^{0}(\lambda)}\left\{\frac{\sqrt{6\left(1-v^{2}\right)} b(1+\mu b)+v \sqrt{3} b^{2}}{1+\mu b+b^{2} / 2}\right\} \frac{\left(\bar{\xi} / t_{\mathrm{eff}}\right)}{\left[1-N^{0} / N_{\mathrm{c}}^{0}(\lambda)\right]} \cos \left(\lambda x_{1} / R\right),
\end{aligned}
$$

where

$$
b=\frac{d}{\sqrt{2\left(1-v_{\mathrm{f}}^{2}\right)} R} \lambda^{2} .
$$

The stresses in the inside face sheet $\left(\sigma_{11}^{-}, \sigma_{22}^{-}\right)$are also given by Eq. (4.4) if the "+" in front of the term containing " $\sqrt{3}$ " in each of the two equations is replaced by "-,", e.g. in the second equation $+v \sqrt{3} b^{2}$ is replaced by $-v \sqrt{3} b^{2}$.

For axisymmetric deformations, the Mises yield condition for the face sheets is $\sigma_{\mathrm{e}}=\sigma_{\mathrm{Y}}$, where the effective stress is given by

$$
\sigma_{\mathrm{e}}^{2} \equiv \sigma_{11}^{2}+\sigma_{22}^{2}-\sigma_{11} \sigma_{22}
$$

Upon evaluating $\sigma_{\mathrm{e}}$ for both the outside and inside sheets using Eq. (4.4), we find that the maximum effective stress occurs in the inside sheet at points where $\cos \left(\lambda x_{1} / R\right)=1$, when $\bar{\xi}>0$. The variation at these points of $\sigma_{\mathrm{e}} / \sigma_{\mathrm{c}}(\lambda)$ as a function of $N^{0} / N_{\mathrm{c}}^{0}(\lambda)$ is shown in Fig. 4 for a selection of imperfection levels and for two choices of the core compliance parameter, $\mu$. The lower value of $\mu$ corresponds to that for an optimally designed shell in the mid-range of the load index, while the higher value corresponds so that for a shell designed according to Eqs. (3.7) and (3.8). In computing the results in these figures, $\lambda$ has been chosen as the critical wave number of the perfect shell, $\lambda_{\mathrm{c}}$, given by Eq. (A.16). However, the dependence of the effective stress on $\lambda$ is relatively weak for values in the vicinity of $\lambda_{c}$.

The value of the abscissa, $N^{0} / N_{\mathrm{c}}^{0}(\lambda)$, at which the shell buckles (i.e. $\left(N_{\mathrm{c}}^{0}\right)_{\text {imp }} / N_{\mathrm{c}}^{0}$ taken from Fig. 3 ) is marked by a solid dot on the curve for each imperfection level in Fig. $4 \mathrm{a}$ and $\mathrm{b}$. The significance of these
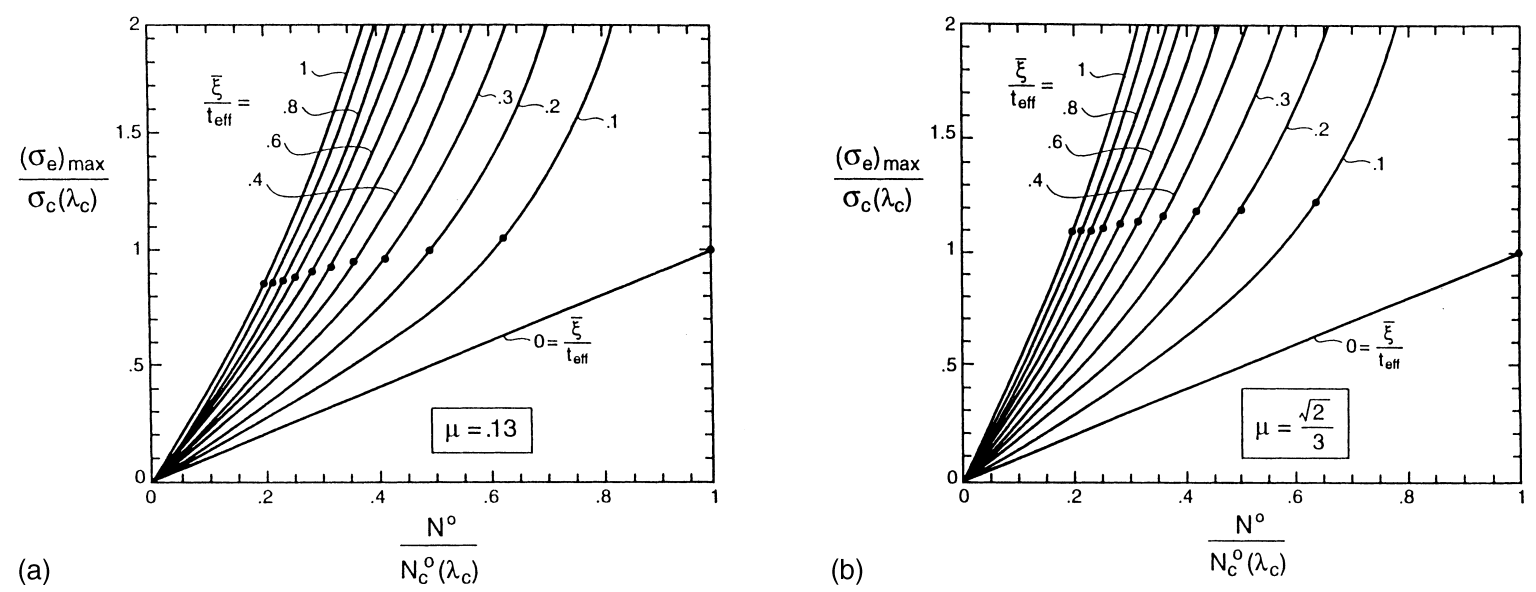

Fig. 4. Maximum effective stress in the face sheets of the imperfect shell as a function of applied load. The effective stress is normalized by the effective stress in the face sheets of the perfect shell at the buckling load, and the applied load is normalized by the buckling load of the perfect shell. On the curve for each imperfection amplitude, the value of the normalized applied load at which the shell buckles is marked by a dot. (a) $\mu=0.13, \lambda=\lambda_{\mathrm{c}}$. (b) $\mu=\sqrt{2} / 3, \lambda=\lambda_{\mathrm{c}}$. 
results for the onset of plastic yielding of the face sheet is as follows. Consider a perfect shell designed according to the procedures of Section 2 such that plastic yielding is coincident with buckling, i.e. $\sigma_{\mathrm{c}}\left(\lambda_{\mathrm{c}}\right)=\sigma_{\mathrm{Y}}$. An imperfect realization of the same shell will experience the onset of yielding in a face sheet when the condition $\sigma_{\mathrm{e}}=\sigma_{\mathrm{Y}}$ is first reached. The point on a given curve in Fig. $4 \mathrm{a}$ and b where $\sigma_{\mathrm{e}} / \sigma_{\mathrm{c}}\left(\lambda_{\mathrm{c}}\right)=1$ provides the value of $N^{0} / N_{\mathrm{c}}^{0}\left(\lambda_{\mathrm{c}}\right)$ at which this condition will be met for a given imperfection level. One notes immediately that the onset of yielding in an imperfect shell occurs at loads above the corresponding load at which that shell will undergo non-axisymmetric buckling in the case where the core shear compliance is not large (Fig. 4a). This means the shell would undergo non-axisymmetric buckling while it is still in the elastic range. Plastic yielding will have no effect on the buckling load of these shells. Imperfect shells with the more compliant cores as shown in Fig. 4b do yield prior to buckling, but only at loads slightly below the predictions for buckling of the elastic shell.

\subsection{Onset of yielding in the core of the imperfect shell}

The optimization of the perfect shell in Section 2 and the study of face sheet yielding of the imperfect shell were carried out under the assumption that the core is elastic. The analysis of the stresses in the imperfect shell just described can be extended to provide the stresses in the core prior to any yielding. In particular, the onset of yielding in the core can be predicted, assuming the face sheets remain elastic.

For the imperfect elastic shell, the stresses in the core are given by

$$
\begin{aligned}
& \frac{\sigma_{11}}{\sigma_{\text {core }}^{\mathrm{c}}(\lambda)}=-\frac{N^{0}}{N_{\mathrm{c}}^{0}(\lambda)}\left\{1-\frac{2 \sqrt{3} b^{2}(z / d)}{1+\mu b+b^{2} / 2} \frac{\left(\bar{\xi} / t_{\mathrm{eff}}\right)}{\left[1-N^{0} / N_{\mathrm{c}}^{0}(\lambda)\right]} \cos \left(\lambda x_{1} / R\right)\right\}, \\
& \frac{\sigma_{22}}{\sigma_{\text {core }}^{\mathrm{c}}(\lambda)}=\frac{N^{0}}{N_{\mathrm{c}}^{0}(\lambda)}\left\{\frac{\sqrt{6\left(1-v^{2}\right)} b(1+\mu b)+v 2 \sqrt{3} b^{2}(z / d)}{1+\mu b+b^{2} / 2}\right\} \frac{\left(\bar{\xi} / t_{\mathrm{eff}}\right)}{\left[1-N^{0} / N_{\mathrm{c}}^{0}(\lambda)\right]} \cos \left(\lambda x_{1} / R\right), \\
& \frac{\sigma_{13}}{\sigma_{\text {core }}^{\mathrm{c}}(\lambda)}=-\frac{N^{0}}{N_{\mathrm{c}}^{0}(\lambda)}\left\{\frac{\sqrt{6\left(1-v^{2}\right)}\left(G_{\mathrm{c}} / E_{\mathrm{c}}\right) \mu b^{2} \lambda}{1+\mu b+b^{2} / 2}\right\} \frac{\left(\bar{\xi} / t_{\mathrm{eff}}\right)}{\left[1-N^{0} / N_{\mathrm{c}}^{0}(\lambda)\right]} \sin \left(\lambda x_{1} / R\right),
\end{aligned}
$$

where $z$ is the coordinate measured along the normal from middle surface, taken positive outward, and

$$
\sigma_{\text {core }}^{\mathrm{c}}(\lambda)=\frac{E_{\mathrm{c}} N_{\mathrm{c}}^{0}(\lambda)}{2 E t}
$$

is the compressive stress in the core of the perfect shell at the eigenload $N^{0}=N_{\mathrm{c}}^{0}(\lambda)$. The wave number $\lambda$ appears as an additional parameter in the core shear stress. The effective stress at any point in the core is

$$
\sigma_{\mathrm{e}}^{2}=\sigma_{11}^{2}+\sigma_{22}^{2}-\sigma_{11} \sigma_{22}+3 \sigma_{13}^{2} .
$$

The maximum effective stress in the core for a representative shell with $\mu=0.13$ and $\lambda=\lambda_{\mathrm{c}}=18.0$ is shown in Fig. 5 as a function of $N^{0} / N_{\mathrm{c}}^{0}(\lambda)$ for various imperfection levels. This shell is the optimally designed shell shown in Fig. 1 with $\eta=0.1$ and $N /\left(E_{\mathrm{f}} R\right)=10^{-5}$; it will also be used as an example in the numerical study in Section 5. The plot has the same form as that employed for face sheet yielding, except that here the effective stress is normalized by the compressive core stress of the perfect shell at buckling. The maximum effective stress in the core is attained at points other than where the normal deflection is maximum or minimum; it has been obtained by examination of all points within the core. The loads at which the shells buckle according to an elastic analysis are again indicated by a solid dot on each curve for each imperfection level. The maximum effective stress in the core for the imperfect shells at buckling is only slightly larger than its value at buckling in the perfect shell, $\sigma_{\text {core }}^{\mathrm{c}}(\lambda)$. It follows that the core will not yield prior to buckling if its yield stress is chosen to be slightly above $\sigma_{\text {core }}^{\mathrm{c}}(\lambda)$. The requirement that the core 


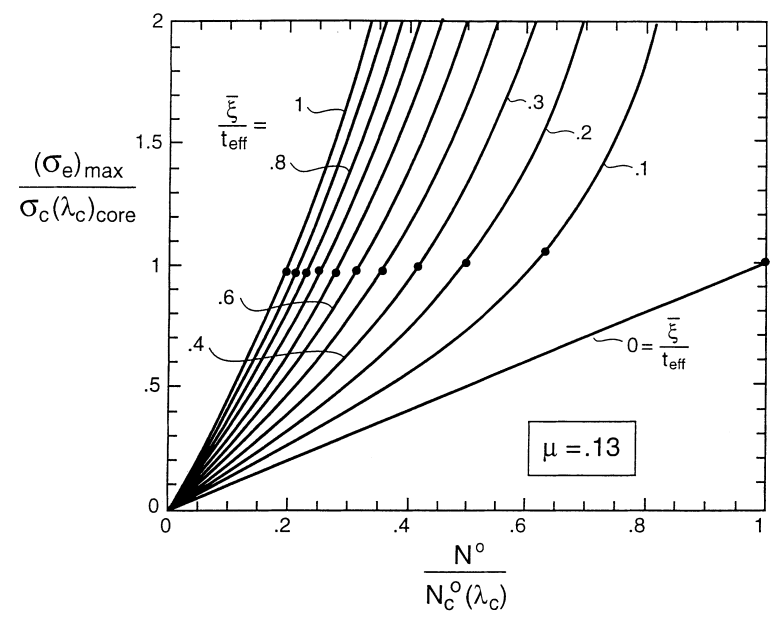

Fig. 5. Maximum effective stress in the core of the imperfect shell as a function of applied load. The effective stress is normalized by the effective stress in the core of the prefect shell at the buckling load, and the applied load is normalized by the buckling load of the perfect shell. On the curve for each imperfection amplitude, the value of the normalized applied load at which the shell buckles is marked by a dot. $\left(\mu=0.13, \lambda=\lambda_{\mathrm{c}}\right)$.

remain elastic until buckling for the perfect shell, with a small extra margin of conservatism, guarantees that the core of the imperfect shell remains elastic prior to buckling.

\subsection{Conclusions from the analytic imperfection studies}

The important conclusion to be drawn from the two parts of the imperfection study is as follows. A sandwich shell optimally designed based on its perfect geometry is not expected to require a significantly larger knock-down factor than that expected for shells buckling elastically, even when, by optimal design, buckling and yielding are coincident in the perfect shell. A similar conclusion was drawn by Hutchinson (1974) for monocoque cylindrical shells under axial compression. At first sight, these findings seem unexpected because the interaction between imperfections and plastic yielding is known to significantly erode the load carrying capacity of columns and plates. The anomaly for the cylindrical shell under axial compression can be attributed to its exceptional geometric imperfection sensitivity in the elastic range. For the shell, the imperfection reduces the overall load at buckling, and therefore the average axial stress, to an extent which offsets the increase in stresses due to bending deflections.

\section{Numerical studies of imperfection sensitivity of optimally designed shells}

A parallel numerical study of the buckling and postbuckling of the imperfect cylindrical shells has also been carried out to substantiate and complement the analytical findings reported above. Shells optimized in Section 3 on the basis of the perfect geometry were considered. In particular, a cylinder from Fig. 1, optimized with fixed relative core density, $\eta=0.1$, and load index, $N /\left(E_{\mathrm{f}} R\right)=10^{-5}$, was chosen for study. The fully dense core material and the face sheet material are taken to be the same aluminum alloy. The face sheet yield stress will be varied to explore its influence on buckling. The elastic modulus of the core is characterized by Eq. (1.1) with $\alpha=1$ and $E_{\mathrm{c}}^{0}=E_{\mathrm{f}} \equiv E$. The other parameters characterizing this shell are

$$
v_{\mathrm{f}}=v_{\mathrm{c}}=0.3, \quad t / R=7.14 \times 10^{-4}, \quad d / R=7.31 \times 10^{-3}, \quad \mu=0.13 .
$$


Recall that the optimal perfect shell is designed such that the face sheets yield at buckling. The compressive yield stress of the core, $\sigma_{\mathrm{Y}}^{\mathrm{c}}$, is the other parameter which will be varied in this study. The core is modeled as an elastic-perfectly plastic material with a Mises yield surface. Plastic compressibility of the metal foam is thus ignored, but this effect is not believed to play a particularly important role in the present application. Subsequent work using a more accurate constitutive characterization of the metal foam may be required to verify this assertion.

A geometric shape imperfection in the location of the middle surface is assumed in the form

$$
\bar{w}=\bar{\xi} \cos \left(\lambda x_{1} / R\right)+\bar{\xi}_{2} \cos \left(\lambda x_{1} / 2 R\right) \cos \left(\lambda x_{2} / 2 R\right),
$$

where $x_{2}$ is the circumferential coordinate. The amplitude of the non-axisymmetric imperfection is fixed at a small fraction of the axisymmetric contribution, $\bar{\xi}_{2}=\bar{\xi} / 10$, in all the calculations presented below. The wave number $\lambda$ is chosen to be that for the critical mode of the perfect shell (A.16), $\lambda_{\mathrm{c}}=18.0$, and the full axial wavelength of the axisymmetric imperfection (A.17) is $\ell=0.35 R$. The non-axisymmetric component of the imperfection is included to trigger non-axisymmetric buckling in the numerical solution process. Note that the wavelengths in the axial and circumferential directions of the non-axisymmetric imperfection are exactly twice that of the axisymmetric component. (The number of the wavelengths around the circumference is 9.) This choice corresponds to the critical combination of modes which couple nonlinearly to give the extreme imperfection-sensitivity of the axially compressed cylinder (Koiter, 1963).

A finite element model of the foam core sandwich shell was formulated using the commercial code ABAQUS. Solutions are sought with periodicity in both the axial and circumferential directions of $2 \ell$. By exploiting symmetry lines of the solution, in addition to the periodicity, one can reduce the region of the shell which is meshed to a square region of dimension $\ell \times \ell$, e.g. $0 \leqslant x_{1} \leqslant \ell, 0 \leqslant x_{2} \leqslant \ell$. Symmetry boundary conditions are imposed on the edges of the cell, and the axial end-shortening is prescribed with the axial load computed as the corresponding work conjugate force quantity. Four-noded, doubly curved layered shell elements are used. The specific designation for the element is S4R. These elements are designed for sandwich construction of the type considered here with relatively low shear compliance in the core. The contributions from the core to the bending and stretching stiffnesses, which were ignored in the analytic part of the study, are accounted for in the finite element model, as is the small bending stiffness contribution of each of the face sheets about their own mid-plane. A convergence study showed that results using a 400element mesh were almost identical to those from an 800 -element mesh. Most of the results presented below have been obtained using the 400-element mesh.

Load-end shortening curves for sandwich shells with elastic cores are shown in Fig. 6 for an imperfection amplitude, $\bar{\xi} / t_{\text {eff }}=0.1$. Here, $\Delta L / L$ is the end shortening per unit length of the shell, $P=2 \pi R N^{0}$, is the total load, $P_{\mathrm{c}}$ is the critical load of the perfect shell. The critical load is $13 \%$ higher than the result based on Eqs. (2.1a) and (2.1b) (i.e. $P_{\mathrm{c}}=1.13 \times 10^{-5}\left(2 \pi R^{2} E\right)$ ), due to the fact that none of the approximations made in simplifying Eqs. (2.1a) and (2.1b) are invoked in the finite element model (e.g. the contribution of the core to the bending and stretching stiffnesses are included and the location of the distance between the face sheet centers is $d+t$ and not $d$ ). Curves 1 and 2 in Fig. 6 show strictly elastic response: curve 1 for the case of an axisymmetric imperfection $\left(\bar{\xi}_{2}=0\right)$ with non-axisymmetric behavior suppressed, and curve 2 for the imperfection (5.2) showing the distinct departure from the axisymmetric response at $P / P_{\mathrm{c}}=0.6$. Curves 3-6 show the influence of the face sheet yield stress. The choice $\sigma_{\mathrm{Y}} / E=0.00791(13 \%$ higher than $\left.\sigma_{\mathrm{Y}} / E=0.007\right)$ corresponds to the optimum design of the perfect shell where the stress in the face sheets at buckling coincides with the yield stress. It is clear in this case that plasticity only erodes the load carrying capacity after the shell has begun to buckle and after the peak load has been attained. Lower values of the face sheet yield stress do result in a lowering of the peak load. The results are completely in accord with the analytical study in Section 3.1.

Load-end shortening curves for a shell with a somewhat larger imperfection are shown in Fig. 7, again, for the case where the core is elastic. Included in this figure is the development of the non-axisymmetric 


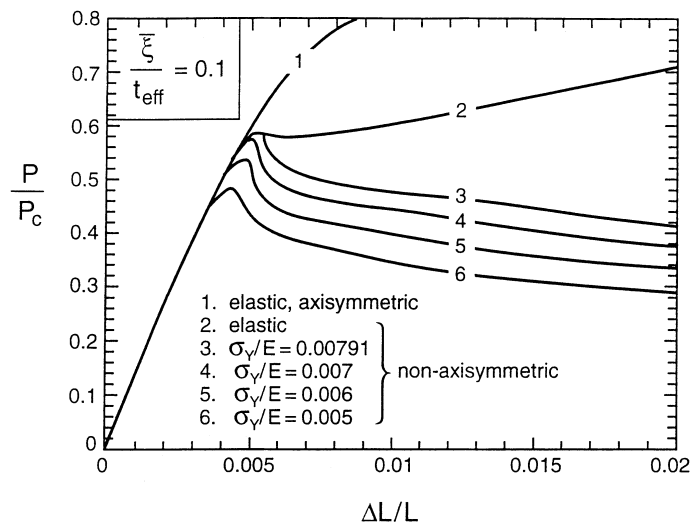

Fig. 6. Effect of face sheet yield stress on load versus end shortening for cylinders with $\bar{\xi} / t_{\mathrm{eff}}=0.1$. Curve 1 is the axisymmetric solution with non-axisymmetric bifurcation excluded. The other curves have a very small non-axisymmetric imperfection component to trigger the non-axisymmetric response. Curve 2 applies to the case where plastic yielding is suppressed. The yield stress of the face sheets for the other curves are indicated. Curve 3 is the case where the yield stress of the face sheet coincides with the face sheet stress at buckling for the perfect shell. The shell parameters are given in Eq. (5.1). The core is elastic.

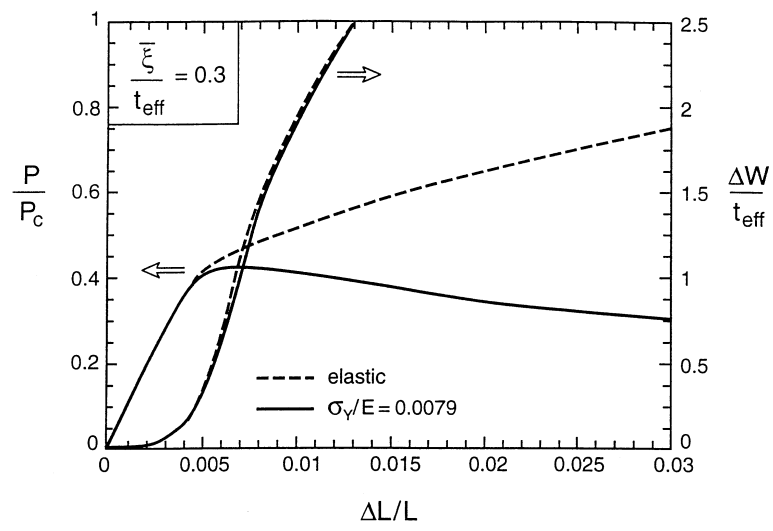

Fig. 7. Load versus end shortening for both an elastic face sheets and face sheets with yield stress equal to the stress in the sheets at the buckling load of the perfect shell. Also shown is the development of the amplitude of the non-axisymmetric deflection, $\Delta w$, . The definition of the buckling load of the imperfect shells used here is that load associated with attainment of $\Delta w / t_{\text {eff }}=1 / 2$. The shell parameters are given in Eq. (5.1) and $\bar{\xi} / t_{\text {eff }}=0.3$. The core is elastic.

response as measured by the difference between the largest and smallest values of the normal deflection in the computational cell, $\Delta w$, around the circumference. At this imperfection level, the load of the elastic shell does not have a local maximum as the non-axisymmetric deflections develop. This behavior is consistent with that documented for the elastic monocoque cylindrical shell by Budiansky and Hutchinson (1972): non-axisymmetric buckling from the axisymmetric state takes place under falling load for small imperfection amplitudes and under gradually increasing load for larger amplitudes. In the results which follow for the effect of the imperfection amplitude on the buckling load, buckling is defined as the load at which $\Delta w / t_{\text {eff }}=0.5$.

Buckling loads are plotted as a function of the normalized axisymmetric imperfection amplitude, $\bar{\xi} / t_{\text {eff }}$, in Fig. 8 for various levels of the face sheet yield stress, where they can be compared with the result of Koiter (1963) for the elastic monocoque shell. The elastic results for the sandwich shell are consistent with 


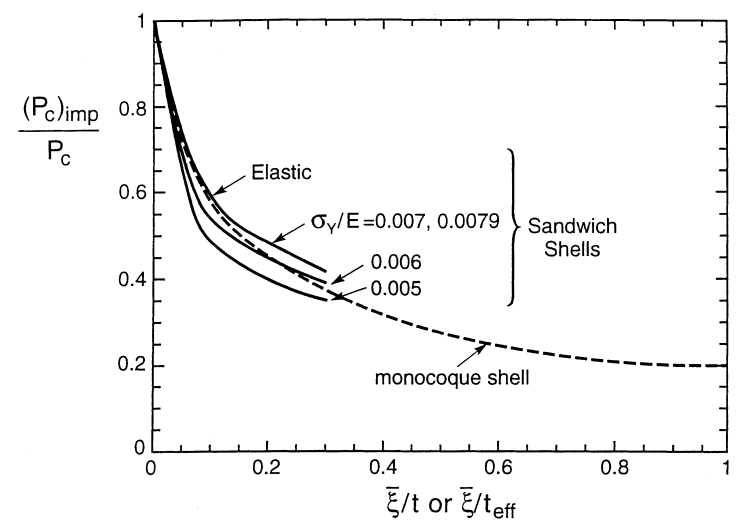

Fig. 8. Influence of face sheet yield stress on the relation between the buckling load and the imperfection amplitude. The amplitude for the imperfection for the elastic monocoque shell is normalized by the shell thickness, while the effective thickness is used for the sandwich shells. Plastic yielding of the face sheets has essentially no effect for yield stresses above $\sigma_{\mathrm{Y}} / E=0.007$. The shell parameters are given in Eq. (5.1). The core is elastic.

those of Tennyson and Chan (1990) in Fig. 3. It is seen that the face sheet yield stresses, $\sigma_{\mathrm{Y}} / E=0.0079$ and 0.007 , have almost no influence on the buckling load. Only when the yield stress is significantly lower than the stress in the face sheets of the perfect elastic shell at buckling is there an appreciable lowering of the knock-down factor below that predicted for elastic shells.

A series of numerical computations carried out to reveal the role of core yielding indicates an even smaller degradation of the knock-down factor than seen for face sheet yielding. The calculations showed that as long as the yield strain of the core, $\sigma_{\mathrm{Y}}^{\mathrm{c}} / E_{\mathrm{c}}$, is greater than about $70 \%$ of the axial strain in the perfect shell at buckling, yielding of the core has almost no effect on the buckling load. The analytical results in Section 3.2 indicate that yielding will start in the core of the imperfect shell at buckling when $\sigma_{\mathrm{Y}}^{\mathrm{c}} / E_{\mathrm{c}}$ is approximately the axial strain in the perfect shell at buckling. It seems plausible that yielding must spread over an appreciable portion of the core before it has any significant effect on the overall behavior. This may be why the core yield strain need not be as large as the analysis of Section 3.2 suggests.

In conclusion, the analytical and numerical studies both suggest that sandwich shells designed on the basis of the perfect geometry should not require larger buckling knock-down factors than elastic shells, even when an optimal design leads to simultaneous buckling and yielding. This conclusion must be tempered by the realization that this study has been restricted to idealized axisymmetric imperfections.

\section{Acknowledgements}

This work was supported in part by a DARPA Multidisciplinary University Research Initiative (ONR Contract N00014-1-96-1028) and in part by the Division of Engineering and Applied Sciences, Harvard University. The authors are indebted to B. Budiansky and A.G. Evans for valuable discussions and to R.C. Tennyson for supplying Fig. 3.

\section{Appendix A. Buckling analysis of sandwich shells}

A brief synopsis of the classical buckling analysis of the axially compressed, perfect sandwich shell is included here. The fact that the lowest buckling load is associated with the axisymmetric mode when the shear compliance of the core is taken into account is attributed to Zahn and Kuenzi (1963) (cf. Tennyson 
and Chan, 1990). For completeness, a compact derivation of this result is given here. The shell theory employed is the Donnell-Mushtari-Wlassow theory extended to include shear of the core. Young's modulus of the core is assumed to be sufficiently low such that the core itself carries a negligible fraction of the resultant membrane stress and bending moment. A parallel development of some of the aspects is given in this article by Tennyson and Chan (1990).

Let $u_{\alpha}$ and $w$ be the tangential $\left(x_{\alpha}\right)$ and normal $\left(x_{3}\right)$ displacements of the middle surface of the shell, and let $\gamma_{\alpha}$ be the components of shear strain in the core in the two tangential directions. Let $\bar{w}$ be the initial geometric imperfection such that $w+\bar{w}$ is the total normal displacement of the middle surface from that of the unloaded perfect shell. The tangential displacements of the outer $(+)$ and inner $(-)$ face sheets are

$$
u_{\alpha}^{+}=u_{\alpha}+\frac{d}{2} \omega_{\alpha}, \quad u_{\alpha}^{-}=u_{\alpha}-\frac{d}{2} \omega_{\alpha}
$$

where $d$ is the separation between the face sheets and $\omega_{1}$ and $\omega_{2}$ are the rotations of the core about the $x_{2}$ and $x_{1}$ axes, respectively. The shear components in the core are given by

$$
\gamma_{\alpha}=\omega_{, \alpha}+\omega_{\alpha} .
$$

The strains of the middle surface are

$$
E_{\alpha \beta}=\frac{1}{2}\left(u_{\alpha, \beta}+u_{\beta, \alpha}\right)+b_{\alpha \beta} w+\frac{1}{2} w,_{\alpha} w,_{\beta}+\frac{1}{2}\left(\bar{w}_{, \alpha} w,_{\beta}+w,_{\alpha} \bar{w}_{\beta}\right),
$$

where the components of the curvature tensor of the undeformed middle surface of the perfect cylindrical shell are $\left(b_{11}=0, b_{22}=1 / R, b_{12}=0\right)$. The resultant membrane stresses are given by

$$
N_{\alpha \beta}=\frac{2 E_{\mathrm{f}} t}{\left(1-v_{\mathrm{f}}^{2}\right)}\left[\left(1-v_{\mathrm{f}}\right) E_{\alpha \beta}+v_{\mathrm{f}} E_{\gamma \gamma} \delta_{\alpha \beta}\right]
$$

and the bending moments are

$$
M_{\alpha \beta}=\frac{E_{\mathrm{f}} t d^{2}}{2\left(1-v_{\mathrm{f}}^{2}\right)}\left[\left(1-v_{\mathrm{f}}\right) K_{\alpha \beta}+v_{\mathrm{f}} K_{\gamma \gamma} \delta_{\alpha \beta}\right],
$$

where

$$
K_{\alpha \beta}=\frac{1}{2}\left(\omega_{\alpha, \beta}+\omega_{\alpha, \beta}\right) .
$$

The stresses in the outside face sheet are given by $\sigma_{\alpha \beta}^{+}=\left(N_{\alpha \beta} / 2+M_{\alpha \beta} / c\right) / t$, whereas the stresses in the inside sheet are $\sigma_{\alpha \beta}^{-}=\left(N_{\alpha \beta} / 2-M_{\alpha \beta} / c\right) / t$. The resultant transverse shear stresses are related to the shear strains in the core by

$$
Q_{\alpha}=G_{\mathrm{c}} d \gamma_{\alpha} .
$$

Equilibrium equations are derived from the principle of virtual work through the assumed strain displacement equations:

$$
\begin{aligned}
N_{\alpha \beta, \beta} & =0, \\
Q_{\alpha, \alpha}+N_{\alpha \beta}\left(-b_{\alpha \beta}+w_{, \alpha \beta}+\bar{w}_{, \alpha \beta}\right) & =0, \\
M_{\alpha \beta, \beta}-Q_{\alpha} & =0 .
\end{aligned}
$$

\section{A.1. The classical buckling problem for the perfect shell $(\bar{w}=0)$}

The inplane equilibrium equations in (the first equation in Eq. (A.8)) can be satisfied by an Airy stress function $\Phi$ with $N_{11}=\Phi_{, 22}, N_{22}=\Phi,,_{11}, N_{12}=-\Phi,,_{12}$. The prebuckling solution for the infinitely long perfect shell is a state uniaxial compression with $N_{11}=-N^{0}$. The equation of inplane compatibility and the re- 
maining equilibrium equations are linearized about the uniform prebuckling state and expressed in terms of $\Phi, w$ and $\omega_{\alpha}$. The resulting buckling equations are

$$
\begin{aligned}
\frac{1}{2 E_{\mathrm{f}} t} \nabla^{4} \Phi-\frac{1}{R} w, 11 & =0, \\
G_{\mathrm{c}} d\left[\nabla^{2} w+\omega_{\alpha, \alpha}\right]-\frac{1}{R} \Phi,{ }_{11}-N^{0} w_{, 11} & =0, \\
\frac{2 E_{\mathrm{f}} t d^{2}}{8\left(1-v_{\mathrm{f}}^{2}\right)}\left[\left(1-v_{\mathrm{f}}\right) \nabla^{2} \omega_{\alpha}+\left(1+v_{\mathrm{f}}\right) \omega_{\beta, \beta \alpha}\right]-G_{\mathrm{c}} d\left[w,_{\alpha}+\omega_{\alpha}\right] & =0 .
\end{aligned}
$$

The eigenvalue is $N^{0}$. Periodic solutions to Eq. (A.9) are sought in the form

$$
\begin{aligned}
w & =\hat{w} t \cos \left(\lambda_{1} x_{1} / R\right) \cos \left(\lambda_{2} x_{2} / R\right), \\
\Phi & =\hat{\Phi} E_{\mathrm{f}} t R^{2} \cos \left(\lambda_{1} x_{1} / R\right) \cos \left(\lambda_{2} x_{2} / R\right), \\
\omega_{1} & =\hat{\omega}_{1}(t / R) \sin \left(\lambda_{1} x_{1} / R\right) \cos \left(\lambda_{2} x_{2} / R\right), \\
\omega_{2} & =\hat{\omega}_{2}(t / R) \cos \left(\lambda_{1} x_{1} / R\right) \sin \left(\lambda_{2} x_{2} / R\right) .
\end{aligned}
$$

Algebraic reduction of Eq. (A.9) provides the equation for the eigenvalue

$$
\frac{N^{0} \sqrt{2\left(1-v_{\mathrm{f}}^{2}\right)} R}{E_{\mathrm{f}} t d}=\frac{2}{b_{1}}+\frac{b_{1}}{\left(1+\mu b_{2}\right)},
$$

where

$$
\begin{aligned}
& \mu=\frac{E_{\mathrm{f}} t}{\sqrt{2\left(1-v_{\mathrm{f}}^{2}\right)} G_{\mathrm{c}} R}, \\
& b_{1}=\frac{d}{\sqrt{2\left(1-v_{\mathrm{f}}^{2}\right)}} \frac{\left(\lambda_{1}^{2}+\lambda_{2}^{2}\right)^{2}}{\lambda_{1}^{2}}, \quad b_{2}=\frac{d}{\sqrt{2\left(1-v_{\mathrm{f}}^{2}\right)}}\left(\lambda_{1}^{2}+\lambda_{2}^{2}\right) .
\end{aligned}
$$

To establish the fact that an axisymmetric mode $\left(\lambda_{2}=0\right)$ gives rise to the lowest eigenvalue and thus the critical buckling load, rewrite Eq. (A.11) as

$$
\frac{N^{0} \sqrt{2\left(1-v_{\mathrm{f}}^{2}\right)} R}{E_{\mathrm{f}} t d}=\frac{2}{b_{1}}+\frac{b_{1}}{\left(1+\mu b_{1}\right)}+\left[\frac{b_{1}}{\left(1+\mu b_{2}\right)}-\frac{b_{1}}{\left(1+\mu b_{1}\right)}\right] .
$$

It is a simple matter to show that the sum of two terms within the square brackets on the right hand side of this equation is non-negative, vanishing if and only if $b_{1}=b_{2} \equiv b$, i.e. when $\lambda_{2}=0$. It follows immediately that the minimum eigenvalue is associated with an axisymmetric mode. When Eq. (A.14) is minimized with respect to $b$ (with $b_{1}=b_{2} \equiv b$ ), one obtains $b=\sqrt{2} /(1-\sqrt{2} \mu$ ) and

$$
\frac{N_{\mathrm{c}}^{0}}{E_{\mathrm{f}} R}=\frac{2 t d}{\sqrt{1-v_{\mathrm{f}}^{2}} R^{2}}\left[1-\frac{\mu}{\sqrt{2}}\right],
$$

which is Eq. (2.1a). The associated critical axisymmetric wave number is

$$
\lambda_{1} \equiv \lambda_{\mathrm{c}}=\left[\frac{2 \sqrt{\left(1-v_{\mathrm{f}}^{2}\right)}}{1-\sqrt{2} \mu} \frac{R}{d}\right]^{1 / 2},
$$

and the wavelength of the critical mode is therefore 


$$
\ell_{\mathrm{c}}=\frac{2 \pi R}{\lambda_{\mathrm{c} 1}}=2 \pi\left[\frac{1-\sqrt{2} \mu}{2 \sqrt{\left(1-v_{\mathrm{f}}^{2}\right)}}\right]^{1 / 2} \sqrt{R d}=2 \pi\left[\frac{1-\sqrt{2} \mu}{6 \sqrt{\left(1-v_{f}^{2}\right)}}\right]^{1 / 2} \sqrt{R t_{\mathrm{eff}}},
$$

where $t_{\text {eff }}$ is defined in Eq. (4.3).

\section{References}

Agarwal, B.L., Sobel, L.H., 1977. Weight comparisons of optimized stiffened, unstiffened, and sandwich cylindrical shells. AIAA J. 14, 1000-1008.

Allen, H.G., 1969. Analysis and Design of Structural Sandwich Panels. Pergamon Press, Oxford.

Budiansky, B., 1999. On the minimum weight of compression structures. Int. J. Solids Struct. 36, 3677-3708.

Budiansky, B., Hutchinson, J.W., (Eds.), 1972. Buckling of circular cylindrical shells under axial compression. Contributions to the Theory of Aircraft Structures. Delft University Press, Netherlands, pp. 239-260.

Evans, A.G., Hutchinson, J.W., Ashby, M.F., 1998. Multifunctionality of cellular metal systems. In: Ashby, M.F., et al. (Eds.), Progress in Materials Science 43, 171-221.

Gibson, L.J., Ashby, M.F., 1997. Cellular Solids: Structure and Properties, Second Ed.. Cambridge University Press, Cambridge, UK. Hutchinson, J.W., 1974. Plastic buckling. Adv. Appl. Mech. 14, 67-144.

Koiter, W.T., 1945. Over de stabiliteit van het elastisch evenwicht (On the Stability of Elastic Equilibrium). Thesis Delft, H. J., Paris, Amsterdam (English translation issued as NASA TT F-10, 833, 1967).

Koiter, W.T., 1963. The effect of axisymmetric imperfections on the buckling of cylindrical shells under axial compression. Koninkl. Nederl. Akademie van Wetenschappen, Ser. B 66, 265-279.

NASA, 1965. Buckling of thin-walled circular cylinders. SP-8007 (revised in 1968).

Sugimura, Y., Meyer, J., He, M.Y., Bart-Smith, H., Grenestedt, J., Evans, A.G., 1997. On the mechanical performance of closed cell Al alloy foams. Acta Mater. 45, 5245-5259.

Sullins, R.T., Smith, G.W., Spier, E.E., 1969. Manual for structural stability analysis of sandwich plates and shells. NASA CR-1457.

Tennyson, R.C., Chan, K.C., 1990. Buckling of imperfect sandwich cylinders under axial compression. Int. J. Solids Struct. 26, 10171036.

Zahn, J.J., Kuenzi, E.W., 1963. Classical buckling of cylinders of sandwich construction in axial compression - orthotropic cores. US Forest Service Note, FPL-018. 